\title{
QUEEN VERSUS ROOK AND PAWN
}

\author{
Lars Rasmussen
}

Tranbjerg, Denmark

Recently, I have been requested to construct a database on Queen versus Rook and Pawn (KQKRP). My work was initiated by a study-solving contest in Skakbladet. One of the studies presented was by Yuri Bazlov, first prize, Chervony Girnik 1984 (see Diagram 1).

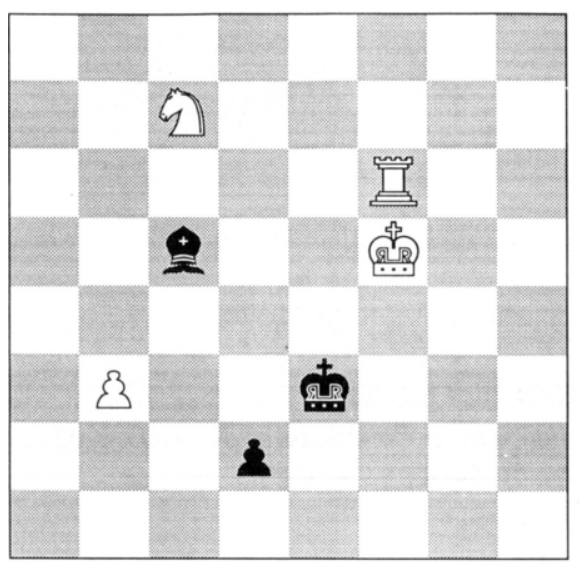

The published solution was: 1. b4 Bd4 2. Nd5+ Kd3 3 . Nf4+ Kc4 4. Rc6+ Kb5 5. Rd6 d1=Q 6. Ke4 Kc4 7. Rc6+ Kxb4 8. Rc1 Qg4 9. Rc4+ Kxc4 stalemate. Some of the contestants pointed out that Black can play: 1. ... d1=Q! 2. bxc5 Qf3+ 3. Ke5 Qg3+ 4. Kd5 Qxc7, rendering it doubtful whether the position was in effect drawn.

Diagram 1: White to Move Draws?

The database was to decide on the matter: what was the correct outcome after 4. ... Qxc7. Since time was very short, I found myself constraint to ignore the endgames arising after promotion of the Pawn. For convenience of reporting the strongest side has been relabeled White so that KQKRP was in fact to be constructed. Failing analysis of the promotion I arbitrarily declared the game to be drawn when Black can promote his Pawn without White being able to capture the promotee (leading to a winning position) in the very first subsequent ply.

This, of course, places additional artificial constraints on White's choice of moves; as a consequence the database thus constructed records some winning positions wrongly as draws. This drawback did not prevent the database from demonstrating Bazlov's study to be a win for Black in that author's terms. The demonstration ran as follows:

5. c6 Qd8+ 6. Rd6 Qa5+ 7. Ke6 Qc7 8. Kd5 Kd3 9. Kc5+ Kc3 10. Rd7 Qa5+ 11. Kd6 Kc4 12. c7 Qa3+ 13. Ke6 Qa8 14. Re7 Qc6+ 15. Kf7 Kd5 16. Kg8 Kd6 17. Rf7 Qa8+ 18. Kh7 Qc8 19. Kg7 Qe8 20. Kf6 Qh8+ 21. Kf5 Qh5+ 22. Kf6 Qh6+ 23. Kf5 Qe6+ 24. Kg5

Black's manoeuvre was not intended to capture the Rook (24. ... Qxf7? 25. c8=Q), but to force White to give up control of the $7^{\text {th }}$ rank.

24. ... Ke5! 25. Kh4 Qh6+ 26. Kg3 Qg6+ 27. Kh2 Qg8 28. Re7+ Kd6 29. Rf7 Ke6 30. Rf2

After 30. Rg7 Qh8+ the Rook will be captured with check.

30. ... Qh7+ 31. Kg3 Qxc7+, and the capture of the Rook in KQKR endgame resulting, will take another 20 moves.

Hence, the database proved that the published draw by stalemate was in fact a win for Bazlov's Black.

As a curiosity arising out of the same database, we present a KQKRP position where 80 moves are required to win (subject to an incomplete analysis of converted positions leaving some doubt as to its optimality). The position quoted is given in Diagram 2.

As long as White's King is this far from the Queenside, he cannot allow Black to move the Pawn. Therefore he must check with the Queen until a position arises where the Pawn temporarily cannot move. Then White moves his King one square, and the whole procedure is repeated. After 27 moves the King arrives at f6, and now Black gains control of the e-file with his Rook to prevent White's King from getting closer. After another 26 moves a position arises which can be found in Chéron (1969) as no. 1445c, and which is wrongly asserted to be a draw by that author. Twelve moves later, notwithstanding, White has achieved Chéron's winning position no. $1445 \mathrm{~b}$. 
1. Qb7+ Kc1 2. Qh1+ Kb2 3. Qh2+ Kb3 4. Qb8+ Kc2 5. Qd6 Rb5 6. Qc6 Rb4 7. Qe4+ Kb3 8. Qb1+ Kc3 9. Qa2 Rb3 10. Qa4!

After 10. Kg5? Kb4 White cannot prevent 11. ... c3 and the game is drawn.

10. ... Rb4 11. Qa3+ Rb3 12. Qc5 Rb4 13. Kg7!!

The only winning move, see the comment to Black's $18^{\text {th }}$ move.

13. ... Kb3 14. Qd5 Ka3 15. Qc6 Kb3 16. Qe6 Ka3 17. Qe3+ Kb2 18. Qe4 Ra4!

If White had played $13 . \mathrm{Kg} 5$ ? or $13 . \mathrm{Kg} 6$ ?, Black could hold on to the draw with a check followed by 19 .... c3.

18. ... Ra4! contains a trap: 19. Kf6? c3!! 20. Qxa4 c2 draw. Not so, however, after 19. ... Ra6+? 20. Ke5 c3 21. Qb7+ Kc1 22. Qxa6 c2 23. Qa2! Kd1 24. Kd4! c1=Q 25. $\mathrm{Kd} 3$ allowing White to preserve the win.

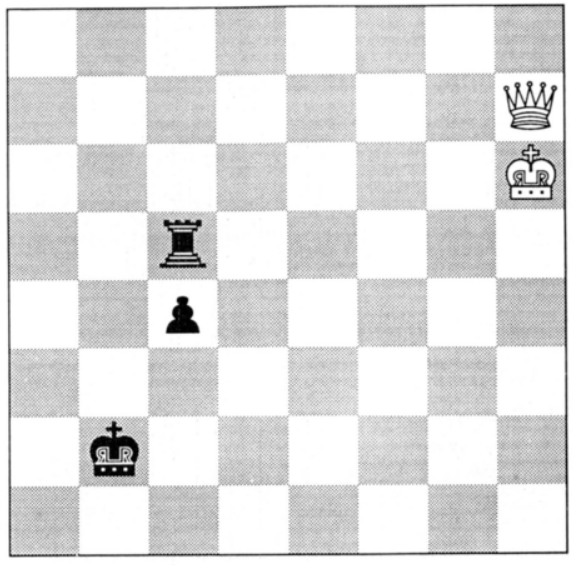

Diagram 2: White to Move.

19. Qe2+! Kc1 20. Qe3+ Kc2 21. Qd4 Rb4 22. Qe4+ Kb3 23. Qb1+ Kc3 24. Qa2 Kd3!

24. ... Rb3?! (as in move 9) does not work here, since White's King is too close now: 25. Kf6! Kb4 26. Ke5, and Black must play 26. ... Rd3, because 26. ... c3? is met by 27. Kd4 Ra3 28. Qc4+ Ka5 29. Kc5.

25. Qa3+ Rb3 26. Qa6 Rb2 27. Kf6 Re2 28. Qb5 Kd4 29. Qa4 Re1 30. Kg5!

Square $\mathrm{f5}$ is reserved for the Queen, see the comment to Black's $31^{\text {st }}$ move.

30.... Kd3 31. Qd7+ Kc3.

If White had played 30. Kf5, Black could have played 31... Kc2 thus avoiding blocking his Pawn, but in this position it is countered by 32. Qf5+ Kd2 33. Qd5+ Kc3 34. Qa5+.

32. Kf4 Re2 33. Qa4 Kd3 34. Qd1+ Rd2 35. Qb1+ Kd4 36. Qb4 Rf2+ 37. Kg3 Ra2 38. Kf3 Kd3 39. Qd6+ Kc3 40. Ke3 Rb2 41. Qa3+ Rb3 42. Qc1+ Kb4+ 43. Ke2 Rd3 44. Qg5 Ra3 45. Qg7 Rd3 46. Qb7+ Kc3 47. Ke1 Rd2 48. Qf3+ Rd3 49. Qf6+ Kb3 50. Qb6+ Kc3 51. Qa5+ Kb3 52. Qb5+ Kc3 53. Qa4 Rd5.

This position also arises from Chéron (1969) no. 1445c after 2 moves on each side. Chéron erroneously suggests 54. Qa3+ Kc2 55. Qb4? Re5+ 56. Kf2 c3! mistakenly declaring position 1445c a draw. However, the true lie of the land is otherwise:

54. Qa8! Rd3 55. Qa3+ Kd4.

Now 55. ... Kc2 56. Qb4 Re3+ (56. ... c3?? 57. Qe4!) 57. Kf2 does not lead to draw, because White gains a tempo by attacking the Rook.

56. Qa5 Rb3 57. Kd1 Rb1+ 58. Kc2 Rb3 59. Kc1 Rc3+ 60. Kb2 Rd3 61. Qb6+ Kd5 62. Qb5+ Kd4 63. Ka2 Rb3 64. Qc6 Kc3 65. Qd6.

This position also arises from Chéron (1969) no. 1445b after 1. Qd5-d6.

65. ... Rb2+ 66. Ka3 Rb3+ 67. Ka4 Rb2 68. Qe5+ Kc2 69. Qd4 c3.

So 69 moves had to pass before the Pawn moved for the first time!

70. Qe4+ Kc1 71. Ka3 Kd2 72. Qd4+ Kc2 73. Qc4 Kb1! 74. Qf1+!

74. Qxc3?? Ra2+ 75. Kb3 Ra3+! 76. Kxa3 stalemate.

74. ... Kc2 75. Qe2+ Kc1 76. Qd3 Rc2 77. Qd4 Kb1 78. Qd1+ Rc1 79. Qb3+ Ka1 80. Qa2 mate.

\section{Conclusion}

The value of this analysis is not so much that Chéron was occasionally at fault, rather it is that, in optimal play, it may well happen that the Pawn is immobile for 69 moves in omniscience. It is inhuman to have this long a foresight. Bazlov's study presents fewer problems: it was flawed by $1 . . . \mathrm{d} 1=\mathrm{Q}$ ! being possible without, at the time it was posed, anybody having knowledge of the consequences of the resultant KQKRP endgame.

\section{Reference}

Chéron, A. (1969). Lehr- und Handbuch der Endspiele. Band III, Zweite verbesserte Auflage. Siegfried Engelhardt Verlag, Berlin-Frohnau. 